\title{
Performance Estimation of Interior Permanent-Magnet Brushless Motors Using the Voltage-Driven Flux-MMF Diagram
}

\author{
T. J. E. Miller, Fellow, IEEE, Mircea Popescu, Calum Cossar, and Malcolm McGilp
}

SPEED Laboratory, Department of Electrical Engineering, University of Glasgow, Glasgow G12 8LT, Scotland, U.K.

\begin{abstract}
The flux-magnetomotive force (flux-MMF) diagram, or "energy conversion loop," is a powerful tool for computing the parameters of saturated interior permanent-magnet brushless motors, especially when the assumptions underlying classical $d q$ theory are not valid, as is often the case in modern practice. Efficient finite-element computation of the flux-MMF diagram is possible when the motor current is known a priori, but in high-speed operation the current regulator can lose control of the current waveform and the computation becomes "voltage-driven" rather than "current-driven." This paper describes an efficient method for estimating the motor performance-average torque, inductances-by solving the voltage-driven problem. It presents experimental validation for a two-pole brushless interior permanent-magnet motor. The paper also discusses the general conditions under which this method is appropriate, and compares the method with alternative approaches.
\end{abstract}

Index Terms-Finite-element method, permanent-magnet brushless motors, simulation, torque calculation.

\section{NOMENCLATURE}

$i, v, \psi \quad$ Instantaneous current, voltage, and flux-linkage.

$p \quad$ The operator $d / d t$; or number of pole-pairs.

$\theta \quad$ Rotor angular position, rad.

$L, R, X$ Inductance, resistance, and reactance.

$T \quad$ Torque, N.m.

[ ] An array; or value at previous time-step.

$\Delta t \quad$ Integration time-step, s.

$\omega \quad$ Speed in electrical $\mathrm{rad} / \mathrm{s}$.

$d, q \quad$ (Subscripts) direct and quadrature axes.

\section{INTRODUCTION}

$\mathbf{B}^{\mathrm{n}}$ RUSHLESS permanent-magnet motors are established in a wide range of applications including hybrid vehicles, servo motors, high-efficiency pumping applications and others, while new applications are emerging as a result of their high efficiency, low noise, and controllability, [1], [5]-[7], [10], [12], [14]-[17]. It is well known that when the magnets are embedded inside the rotor iron, as in the "interior permanent-magnet motor" (IPM), calculations based on classical $d q$ theory can be unreliable, mainly because of the variation of parameters due to saturation.

For example $X_{q}$ in such machines can vary by at least $700 \%$ between no-load and full-load, while cross saturation influences the $d$-axis parameters in a complex manner [1]-[7], [9]. The difficulties are increased by the use of fractional-slot windings with small numbers of slots/pole (even less than 1), non-circular laminations, and other departures from the ideal machine, so that neither the EMF waveform nor the variation of inductance with rotor position is sinusoidal.

Digital Object Identifier 10.1109/TMAG.2006.874512
Most previous analyses rely on the classical $d q$ model [1]-[7], [9], and although they allow for cross-saturation effects they restrict themselves to steady-state operation and ignore the effect of rotor position on the $d q$-axis parameters.

As long as the current waveform is known a priori, these problems can be overcome in an efficient computation in which the finite-element method is used to determine one cycle of the flux-linkage waveform, which is then plotted against the current waveform to establish a "loop" whose area gives the average electromagnetic torque. This loop diagram is known as the "flux-MMF diagram" or "i-psi loop" or "energy conversion loop" [9]. Bearing in mind that finite-element formulations are fundamentally always "current-driven," the computation is efficient because of the foreknowledge of the current waveform, so that a series of current values can be used as inputs to the finite-element process. This method does not necessarily rely on $d q$ theory.

At higher speeds it is normal for the current-regulator to lose control of the current waveform, and ultimately at the highest speeds the drive may operate in six-step mode. In this case, the applied voltage waveform is known but the current waveform is not. Similarly, if the machine is generating into a rectifier, the applied voltage at the machine terminals is known or calculable, whereas the current waveform is unknown.

The accuracy of the finite-element method is very desirable in design work, but the problem is how to get quick results, especially in solving the "voltage-driven" problem. Certain finite-element programs can incorporate differential electric-circuit equations in their solvers, but the solution time is a serious problem because it must run out over many cycles until a steady state is achieved. Some finite-element programs can be coupled to general-purpose system simulation software. When such general-purpose tools are used, the setup time may be an additional issue. The objective here is to develop a special-purpose method which is specifically designed for rapid computation, and to throw some light on the actual need for voltage-driven finite-element solutions. 


\section{Mathematical Model}

\section{A. The Problem to be Solved}

The basic problem is to solve the voltage equations of the machine and its drive. For a three-phase machine, the voltage equations of the individual phases are

$$
\begin{aligned}
& v_{a}=R i_{a}+p \psi_{a} ; \\
& v_{b}=R i_{b}+p \psi_{b} ; \\
& v_{c}=R i_{c}+p \psi_{c} .
\end{aligned}
$$

It is also necessary to know the magnetization curves, i.e., the relationship between the flux-linkages $\psi$ and the currents $i$ :

$$
\begin{aligned}
& \psi_{a}=\psi_{a}\left(i_{a}, i_{b}, i_{c}\right) ; \\
& \psi_{b}=\psi_{b}\left(i_{a}, i_{b}, i_{c}\right) ; \\
& \psi_{c}=\psi_{c}\left(i_{a}, i_{b}, i_{c}\right) .
\end{aligned}
$$

When a simulation program is used to solve (1), the voltages are known and the currents are unknown. A "time-stepping" solution proceeds by integrating (1) step-by-step, and from the new flux-linkages at the end of each time-step the currents must be obtained by inverting (2), ready for the next time-step. Sometimes (2) is expressed in the form

$$
[\psi]=\left[L\left(\theta, i_{a}, i_{b}, i_{c}\right)\right][i]
$$

so that the necessary inversion is a simple matrix operation. Unfortunately, in all but the simplest cases, the self and mutual inductances in the $[L]$ matrix are complicated functions of rotor position $\theta$. When magnetic saturation occurs, they are also functions of current, and then their definition and computation can become ambiguous and obscure. Sometimes (3) is substituted in (1) ab initio, making currents $[i]$ the state variables instead of flux-linkages $[\psi]$. Since current varies much more rapidly than flux-linkage, it becomes necessary to use a much smaller time-step and the solution becomes much slower.

The finite-element method is fundamentally a method of evaluating (2). Known phase currents $i_{a}, i_{b}, i_{c}$ are expressed as current-density in slot regions, and the unknown flux-linkages $\psi_{a}, \psi_{b}, \psi_{c}$ are computed from line integrals of vector potential along the winding conductors. If the current waveform is given, the finite-element method can be used to compute the flux-linkage waveforms which can be differentiated with respect to time $(p \psi)$ and substituted in (1) to predict the voltage that would be necessary to achieve the assumed current waveforms. This somewhat "back-to-front" process only works with known current waveforms, but it is quick. Rather than providing a full "voltage-driven" solution, it gives a quick "current-driven" solution together with an indication of the required drive voltage.

\section{B. Limitations of Classical Theory}

The classical theory of the synchronous machine is based on the $d q$ transformation, usually in the form attributed to Park [11]. In the ideal case, it eliminates the rotor position $\theta$ from the $[L]$ matrix, producing an immense simplification. For 3-wire connections it reduces the number of currents from 3 to 2 , and likewise for the voltages and flux-linkages.
In its classical form this theory rests on two assumptions: $(a)$ the windings are sinusoidally distributed around the stator periphery, giving rise to a sinusoidal induced voltage and sinusoidal variation of self- and mutual inductance with rotor position; and $(b)$ the magnetic circuit is linear.

If phasors are to be used to describe steady-state operation, it must also be assumed $(c)$ that all currents and voltages have sinusoidal time-waveforms. In modern practice all three of these assumptions are often violated to some degree, in some cases to such an extent that the classical model gives seriously misleading results.

In spite of these limitations it is still desirable to work in $d q$ axes, which are synchronous and fixed to the rotor. This is the natural reference frame for the synchronous machine. Even under nonideal conditions its simplifying properties are valuable. The $d q$ transformation is described in [11]:

$$
\begin{aligned}
\psi_{d}= & \frac{2}{3}\left[\psi_{a} \cos \theta+\psi_{b} \cos (\theta-2 \pi / 3)\right. \\
& \left.+\psi_{c} \cos (\theta+2 \pi / 3)\right] ; \\
\psi_{q}= & -\frac{2}{3}\left[\psi_{a} \sin \theta+\psi_{b} \sin (\theta-2 \pi / 3)\right. \\
& \left.+\psi_{c} \sin (\theta+2 \pi / 3)\right] .
\end{aligned}
$$

The same transformation is used for currents and voltages. When it is substituted in (1), the voltage equations

$$
\begin{aligned}
& v_{d}=R i_{d}+p \psi_{d}-\omega \psi_{q} \\
& v_{q}=R i_{q}+p \psi_{q}+\omega \psi_{d}
\end{aligned}
$$

are obtained. The zero-sequence component is omitted if the machine has no neutral connection.

The transformation is valid even when the idealizations $(a)-(c)$ are not satisfied. In that case it is merely a mathematical mapping; (5) does not inherently require magnetic linearity or sinusoidal winding distributions.

Digital simulation of the machine performance requires the step-by-step integration of (5). For example, by Euler's method the $d$-axis equation becomes

$$
\begin{aligned}
\psi_{d} & =\int\left(v_{d}-R i_{d}+\omega \psi_{q}\right) d t \\
& \approx\left(v_{d}-R i_{d}+\omega \psi_{q}\right) \Delta t+\left[\psi_{d}\right]
\end{aligned}
$$

and similarly for the $q$-axis, where $\left[\psi_{d}\right]$ means the value of $\psi_{d}$ at the previous time-step. At each new time-step, the currents $i_{d}$ and $i_{q}$ must be updated from the new flux-linkages, using the magnetization curves.

\section{Mathematical Form of the Magnetization Curves}

In the classical synchronous machine with sine-distributed windings and no saturation, these relationships are simple straight lines:

$$
\psi_{d}=\Psi_{M}+L_{d} i_{d} ; \quad \text { and } \psi_{q}=L_{q} i_{q}
$$

where $L_{d}$ and $L_{q}$ are the synchronous inductances and $\Psi_{M}$ is a constant flux-linkage produced by the magnet or the fieldwinding, depending on the type of machine. It is trivial to invert (7) at each time-step. In the classical case, the $d$ and $q$ axes 
are uncoupled and the magnetization curves (7) are independent of rotor position $\theta$.

In the general case the windings may not be sine-distributed and the magnetic circuit may be saturable. The magnetization curves then have the general form

$$
\psi_{d}=\psi_{d}\left(i_{d}, i_{q}, \theta\right) \text { and } \psi_{q}=\psi_{q}\left(i_{d}, i_{q}, \theta\right) .
$$

The magnetization curves in this form can be obtained by transformation from finite-element evaluations of (2). In general $\psi_{d}$ is a function not only of $i_{d}$ but also of $i_{q}$ (cross coupling) and even of $\theta$, and likewise $\psi_{q}$ is a function of $i_{d}$ and $\theta$ as well as $i_{q}$. The functional relationships (8) require a vast number of finite-element calculations to define them [2], as well as an appropriate interpolating function. They are much more complex than (7), which have the form

$$
\psi_{d}=\psi_{d}\left(i_{d}\right) \text { and } \psi_{q}=\psi_{q}\left(i_{q}\right)
$$

in which there is no cross coupling and no variation with rotor position, and which are easily inverted even when they are nonlinear. One option is to limit the extent of the "saturation model" to this form, implicitly ignoring cross saturation and any variation with rotor position [1], [3], [4]. Thus, the influence of of the direct axis current, $i_{d}$ on the q-axis flux-linkage $\psi_{q}$ is negligible most of the interior PM motors [6].

In (8), the functional influence of $\theta$ is due to winding harmonics and any variation of magnetic permeance as the rotor rotates. The obvious source of such permeance variation is on the stator. It includes any grain-orientation in the steel; the presence of notches or holes in the lamination, or chamfers and cut-outs at its outer surface; and the effects of slotting.

Even without these effects, and even with sine-distributed windings, if the magnetic circuit is saturable there remains the possibility of a functional influence of $\theta$, induced by the variation of current as the rotor rotates. In well-designed machines the functional influence of $\theta$ in (8) should be weak, otherwise it would be difficult to produce smooth torque. But it requires good calculations to achieve this. With no $\theta$ variation, the magnetization curves have the form

$$
\psi_{d}=\psi_{d}\left(i_{d}, i_{q}\right) \text { and } \psi_{q}=\psi_{q}\left(i_{d}, i_{q}\right)
$$

This is the form presented in [2], but only in the form of graphical data with no attempt to provide the interpolating function that is necessary in connection with the time-stepped solution of the voltage equations.

\section{Methods for Solving the Problem}

In the process of integrating (6), we have seen that "new" values of $\psi_{d}$ and $\psi_{q}$ are generated at each time-step, and that the currents $i_{d}$ and $i_{q}$ must be updated. The integration is computationally fast, and therefore our main concern is with the methods for updating the currents, which can be classified as follows.

A) If there is no magnetic saturation and the windings are sinusoidally distributed, (7) can be used. This is by far the fastest method, but obviously it requires reliable values of $L_{d}, L_{q}$ and $\Psi_{M}$.
B) Method A) can be modified by precalculating a currentdriven $i-\psi$ loop and adjusting the values of $L_{d}, L_{q}$ and $\Psi_{M}$ to take account of saturation and $\theta$ variation in an average sense over one electrical cycle.

C) As the time-stepping solution proceeds, the values of $L_{d}, L_{q}$ and $\Psi_{M}$ can be updated by means of a single-point finite-element calculation at each time-step, or after an arbitrary number of time-steps, and used in (7) to determine the currents at the next time-step.

D) An entire set of magnetization curves can be precalculated in the form of (8); or in the simplified form of (9) (with no cross saturation or $\theta$ variation) or (10) (with cross saturation but no $\theta$ variation). Whatever the form of the magnetization curves, the supposition is that they must be precalculated by finite-elements and expressed in a suitable form for time-stepping simulation. The necessary interpolation of the magnetization curves by a numerical curve-fitting function is not a trivial matter and is likely to introduce additional errors. Moreover, this process implies a separation between the computation of the magnetization curves and their use in the time-stepping simulation, which is very inconvenient from the user's point of view.

E) As the time-stepping simulation proceeds, at each timestep a series of single-point finite-element solutions can be executed under the control of a Newton-Raphson type algorithm which adjusts the currents $i_{d}$ and $i_{q}$ to match the flux-linkages computed by means of the circuit simulation. Assuming that four finite-element solutions are required at each iteration (to provide the necessary elements of the Jacobian), and assuming five iterations per point, this method requires 20 times more finite-element solutions than Method C).

For normal design work these times suggest that methods A) and B) are much to be preferred, especially if their accuracy can be substantiated by intermittent use of Method C). Taken together, these methods are perfectly sufficient for the currentdriven problem. The results presented later suggest that they may also be sufficient for the voltage-driven problem, in which case methods D) and E) appear to be quite uneconomic.

The average electromagnetic torque is obtained from the sum of the loop areas enclosed within the $i-\psi$ locus for each phase, and examples are given in Figs. 4-6, [9]. Method A) is the only one that permits the separation of permanent-magnet alignment torque and reluctance torque. All the others have the possibility of computing torque also by Maxwell stress.

\section{EXPERIMENTAL AND COMPUTATION DATA}

Fig. 1 shows the cross section with flux-lines plot of a twopole brushless IPM motor which is controlled by a PWM inverter. Each magnet pole comprises three separate $\mathrm{NdFeB}$ magnets. The three-phase winding has two pole-groups per phase, each with four coils having turns and coil-pitches of 50/11;46/9; $40 / 7$ and 30/5, respectively. The rotor diameter is $62 \mathrm{~mm}$ and the stack length is $48.6 \mathrm{~mm}$. There is no skew.

Complications are introduced by the complex outer shape of the stator lamination. Thus, although the windings are reasonably sinusoidally distributed, the permeance harmonics result in considerable ripple in the back EMF (see Fig. 2). There is also 


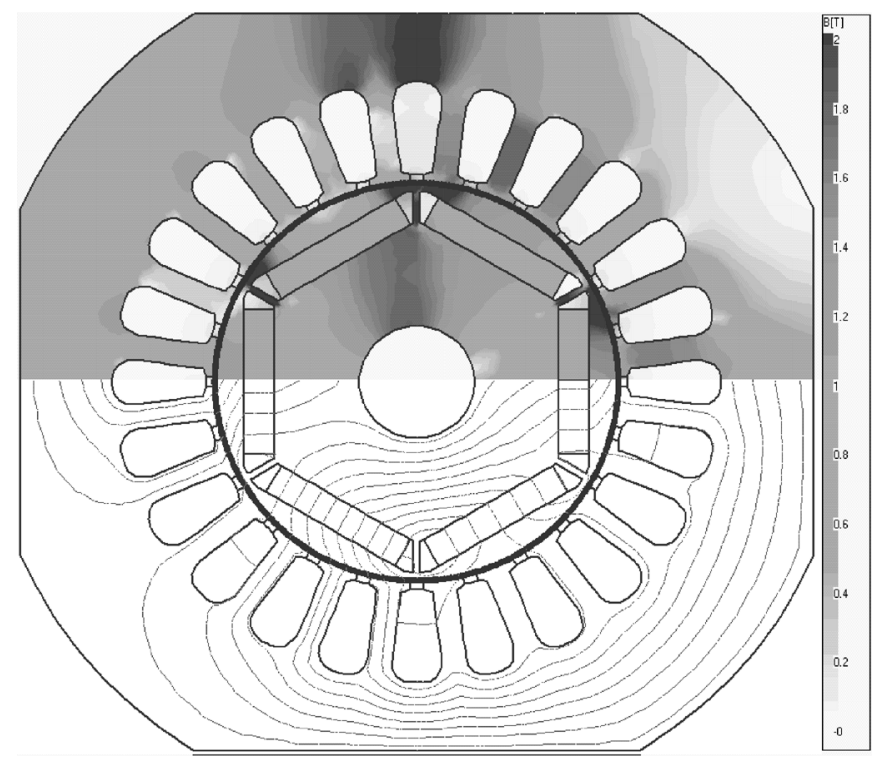

Fig. 1. Cross section of the tested motor with flux-lines plot.

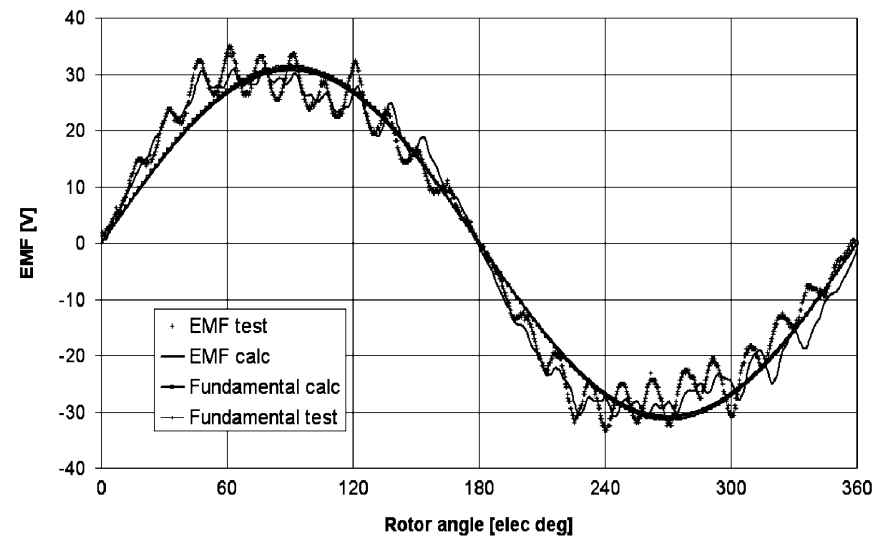

Fig. 2. Open-circuit back EMF.

an asymmetry in the EMF waveform caused by the squared-off shape of the stator lamination and the nonuniform stator yoke section. This affects the three phases differently according to their positions. The importance of the open-circuit waveforms is to show the degree to which the motor might appear to be amenable to analysis by $d q$ theory. In spite of the ripple in the EMF, the sinusoidal shape of the flux-linkage in Fig. 3 suggests that good results should be obtained for the average torque. Note that the flux densities in certain stator yoke regions and rotor bridges are approaching $1.8 \mathrm{~T}$ even in the open-circuit condition, so the machine is partly saturated.

Fig. 4 shows the $i-\psi$ loop diagram for sinewave current control, comparing the measured loop with the finite-element calculation having a peak phase current of $1.23 \mathrm{~A}$ at $440 \mathrm{rpm}$ with no phase advance. The loop torque is $1.10 \mathrm{~N} \cdot \mathrm{m}$ and this agrees almost exactly with the measured value. Both the current and the flux-linkage waveforms are closely sinusoidal in this condition.

Fig. 5 shows a sinusoidal voltage-PWM control in which the switching duty-cycle is modulated to give a sinusoidal terminal voltage (apart from the switching harmonics). Accordingly the flux-linkage is nearly sinusoidal but the current is not perfectly

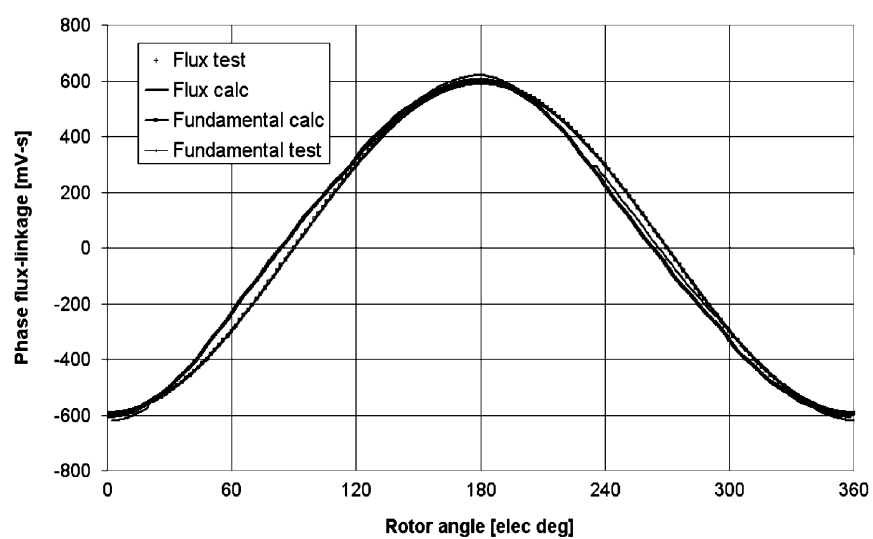

Fig. 3. Flux-linkage per phase.

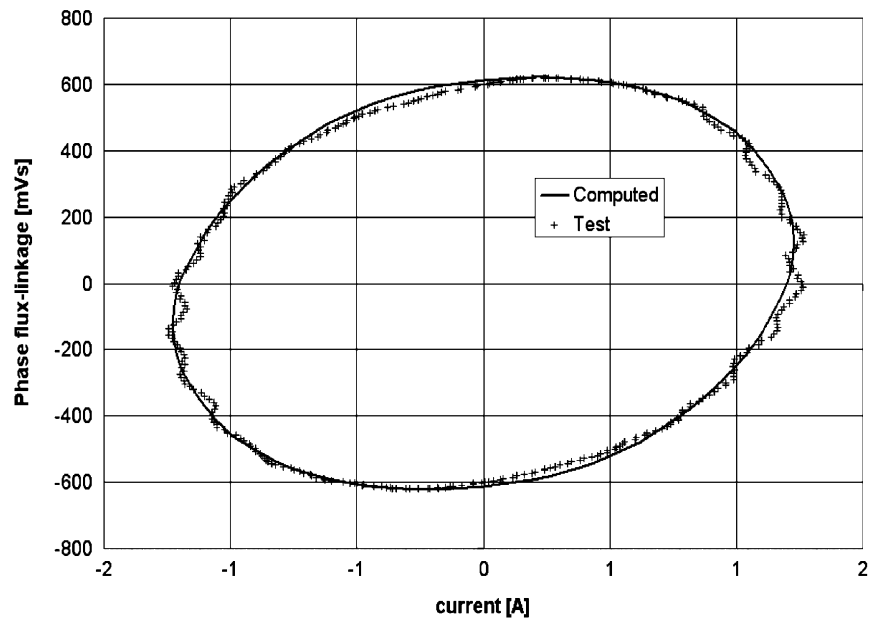

Fig. 4. $i-\psi$ loop for sinewave current control.

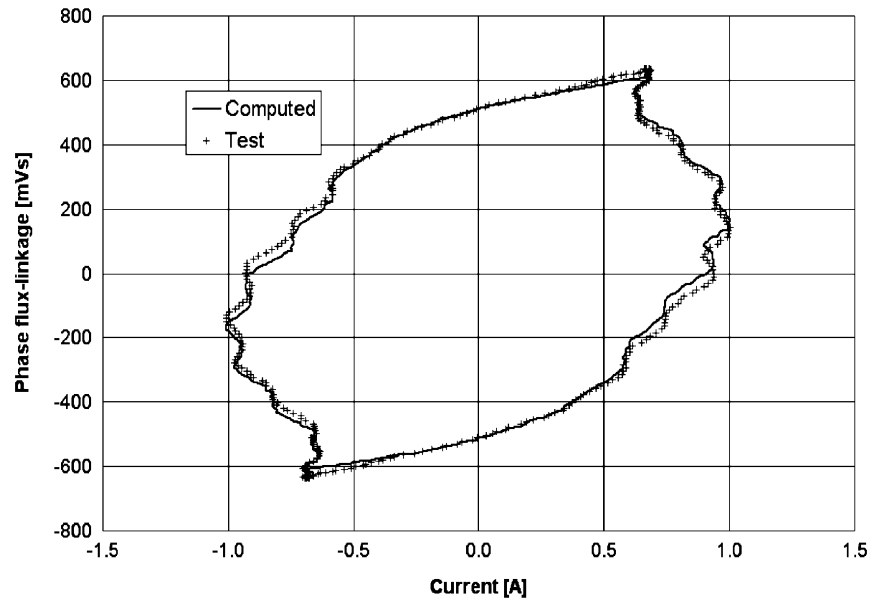

Fig. 5. $i-\psi$ loop for voltage PWM current control.

sinusoidal. The calculated loop in Fig. 5 is obtained by a currentdriven finite-element computation using the measured current waveform, giving a loop torque of $0.79 \mathrm{~N} \cdot \mathrm{m}$ compared with the measured value of $0.76 \mathrm{~N} \cdot \mathrm{m}$.

Fig. 6 shows the $i-\psi$ loop for six-step operation at $528 \mathrm{rpm}$ with a dc link voltage of $70 \mathrm{~V}$. The calculated loop is obtained by a current-driven finite-element computation using the measured current waveform, giving a loop torque of $1.14 \mathrm{~N} \cdot \mathrm{m}$ compared 


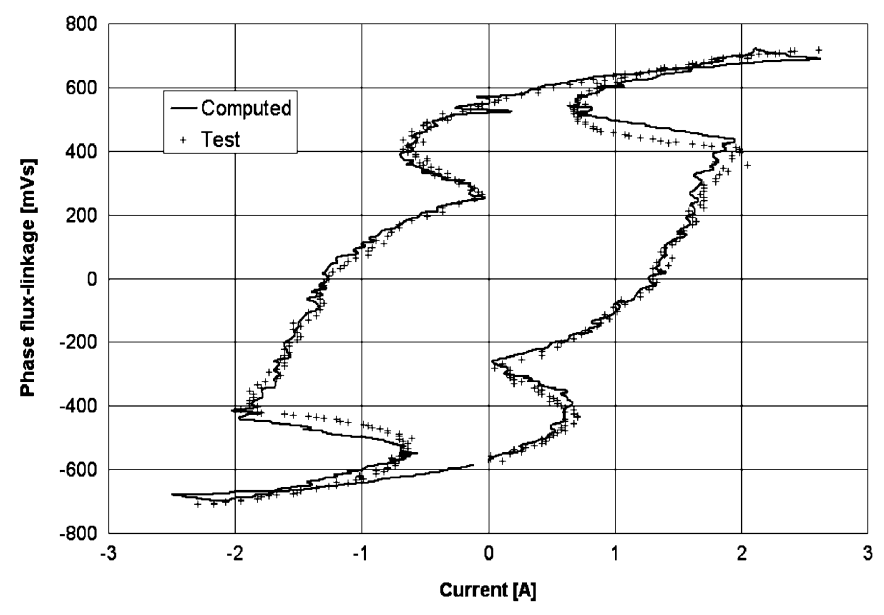

Fig. 6. $i-\psi$ loop for six-step current control.

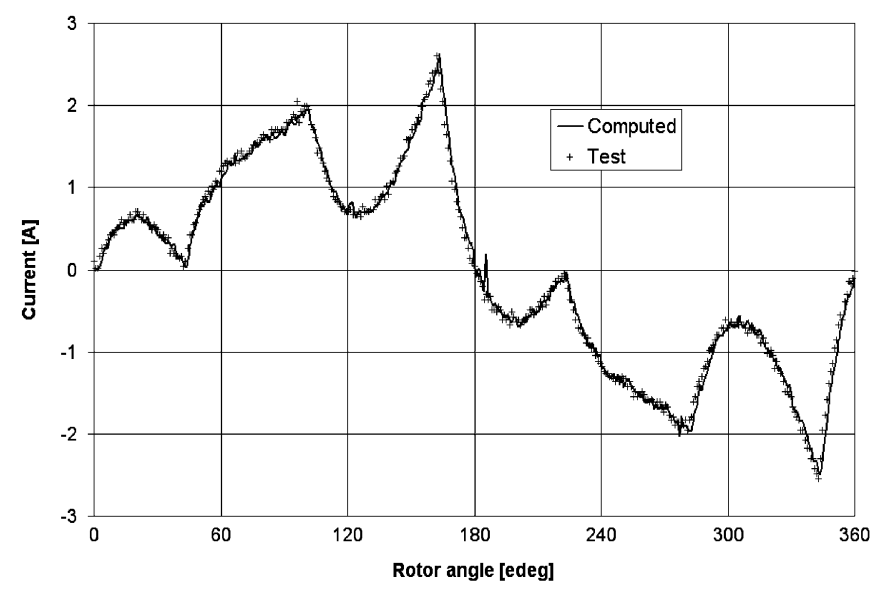

Fig. 7. Phase current for six-step voltage.

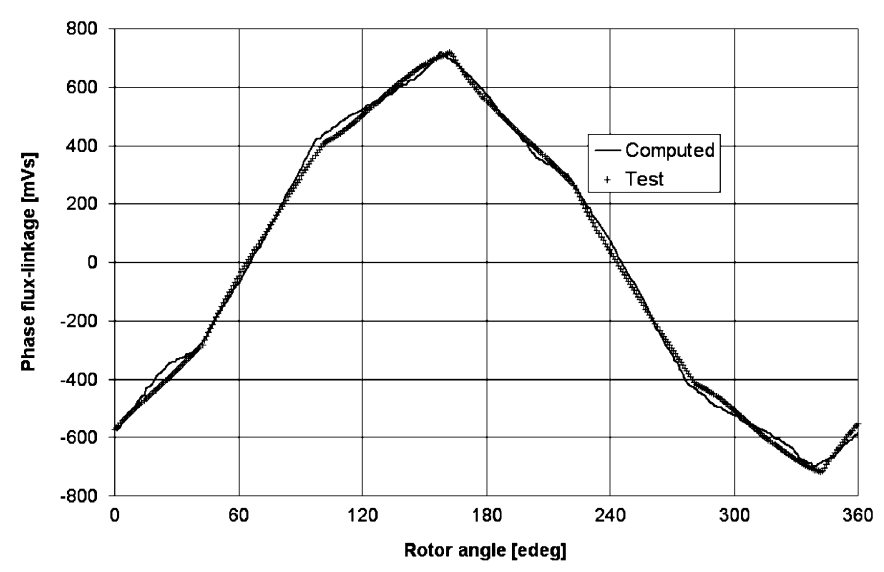

Fig. 8. Phase flux-linkage for six-step voltage.

with the measured value of $1.17 \mathrm{~N} \cdot \mathrm{m}$. The current for this sixstep condition is shown in Fig. 7, and the flux-linkage in Fig. 8.

The flux-linkage waveform consists essentially of straightline segments while the phase voltage waveform can be reconstructed by differentiating this waveform and adding the resistive volt-drop, as shown in Fig. 9 .

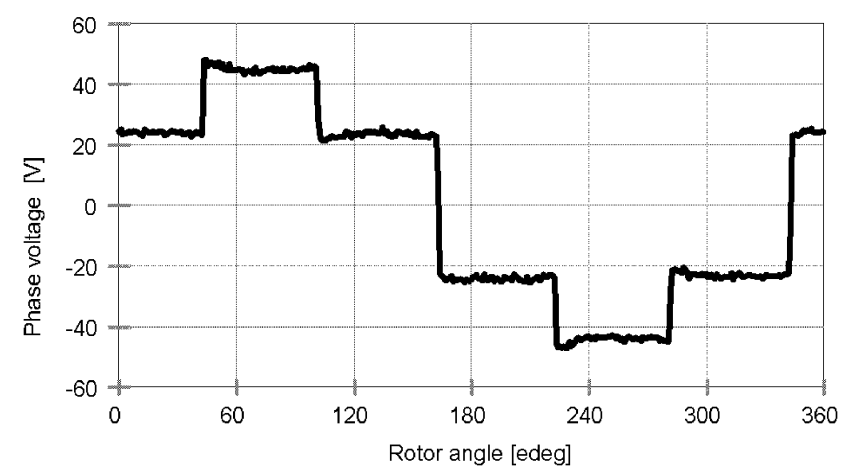

Fig. 9. Phase voltage reconstructed from Figs. 7 and 8.

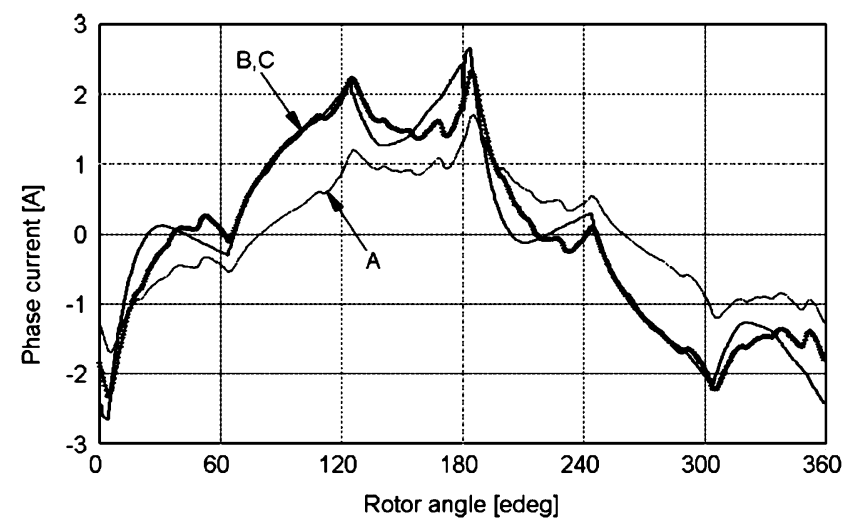

Fig. 10. Computed current for six-step operation.

To illustrate the effectiveness of the computation methods A), B), and C) of Section II, the six-step drive is taken as the clearest example of a case where the applied voltage waveform is known a priori, but the current waveform is not.

Considering Method C) first, Fig. 10 is computed from scratch using motor dimensions and materials, with the time-stepping solution of the differential circuit equations in the $P C-B D C$ program and the values of $L_{d}, L_{q}$, and $\Psi_{M}$ updated by a finite-element calculation at each time-step using [13].

Also shown (dotted) in Fig. 10 is the current computed by Method B) with fixed values of $L_{d}, L_{q}$ and $\Psi_{M}$ after adjusting to match a current-driven finite-element loop calculation at $2.5 \mathrm{~A}$ peak. The adjustments required a $15 \%$ reduction in $d$-axis magnet flux $\Psi_{M}$, a $20 \%$ reduction in $X_{d}$, and a $22 \%$ reduction in $X_{q}$ to allow for the overall saturation at this condition. The loop torque compares closely with that of Method D).

Fig. 10 also shows the current computed by Method A) with fixed unsaturated inductances $L_{d}$ and $L_{q}$ and magnet flux-linkage $\Psi_{M}$ (7). The current is underestimated and so is the torque, even though the flux-linkage waveform is the same. The measured variation of the values of $L_{d}$ and $L_{q}$ with current is illustrated in Fig. 11.

Note the reluctance ratio of $1 / 2$ between $d q$ axis inductances. Also, the same saturation level occurs in both axes, where the corresponding inductance varies with $20 \%$ as compared to the unsaturated value. The measured inductance values are obtained using a Jones bridge test stand [8]. 


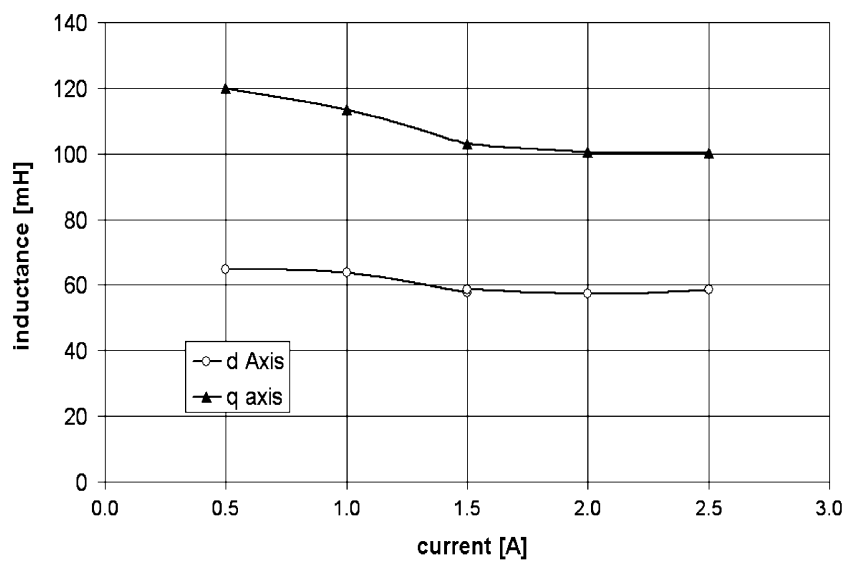

Fig. 11. Measured $d q$ axis inductances.

\section{CONCLUSION}

The flux-MMF diagram may be used to compute the average torque and inductances in an interior permanent-magnet motor. Accurate estimations of the parameters for saturated interior PM motor are obtained by incorporating the finite-element solutions into a $d q$ "voltage-driven" equations system.

It is shown that computation burden economies can be made in the application of the finite-element method to the accurate calculation of the average electromagnetic torque. The paper develops an improved understanding of the extent to which classical $d q$ axis analysis and phasors can be used legitimately in nonsinusoidal current operating conditions such as six-stepping. It also provides a means of testing the rigour of various common assumptions about the motor-for example, whether the synchronous inductances vary with rotor position, or whether the phasor diagram can be used when the current waveform is not sinusoidal.

\section{ACKNOWLEDGMENT}

The authors would like to thank P. Scavenius, N.-C. Weihrauch, and P. Hansen of Danfoss Compressors, Flensburg, Germany, who supplied the special test motor used in this paper. The authors would also like to acknowledge the support of the member companies of the SPEED Consortium and the assistance of P. Miller and I. Young of the SPEED Laboratory.

\section{REFERENCES}

[1] L. Tutelea, M. C. Kim, Y.-D. Chun, T. H. Kim, S.-B. Lim, J. S. Ahn, J. Lee, and I. Boldea, "A set of experiments to more fully characterize linear PM oscillatory machines," IEEE Trans. Magn., vol. 41, no. 10, pp. 4009-4011, Oct. 2005.
[2] G. Štumberger, B. Polajzer, B. Štumberger, M. Toman, and D. Dolinar, "Evaluation of experimental methods for determining the magnetically nonlinear characteristics of electromagnetic devices," IEEE Trans. Magn., vol. 41, no. 10, pp. 4030-4032, Oct. 2005.

[3] N. Bianchi and S. Bolognani, "Magnetic models of saturated interior permanent magnet motors based on finite element analysis," in IEEE Ind. Appl. Soc. Annu. Mtg., Conf. Record, St. Louis, MO, 1998, pp. 27-34.

[4] N. Bianchi and S. Bolognani, "Saturation effect on parameters and voltampere ratings of an interior PM motor for a spindle drive, EPE'99," in Proc. Electrical Drives Design and Applications, Lausanne, Switzerland, 1999.

[5] T. M. Jahns, G. B. Kliman, and T. W. Neumann, "Interior permanentmagnet synchronous motors for adjustable-speed drives," IEEE Trans. Ind. Appl., vol. 22, pp. 738-747, 1986.

[6] D. M. Ionel, M. J. Balchin, J. F. Eastham, and E. Demeter, "Finite element analysis of brushless DC motors for flux weakening operation," IEEE Trans. Magn., vol. 32, no. 5, pt. 2, pp. 5040-5042, Sep. 1996

[7] A. C. Jack and B. C. Mecrow, "Design and initial test results from a permanent magnet synchronous motor for a vehicle drive," in Conf. Rec. ICEM 1992, Manchester, vol. II, pp. 751-755.

[8] T. J. E. Miller, "Methods for testing permanent-magnet AC motors," in IEEE Ind. Appl. Soc. Annu. Mtg. (IAS), Toronto, ON, Canada, Oct. 1981, pp. 494-499.

[9] D. A. Staton, W. L. Soong, R. P. Deodhar, and T. J. E. Miller, “Torque prediction using the flux-MMF diagram in AC, DC and reluctance motors," IEEE Trans. Ind. Appl., vol. 32, no. 1, pp. 180-188, Jan.-Feb. 1996.

[10] M. A. Jabbar, L. Zhejie, and D. Jing, "Time-stepping finite-element analysis for the dynamic performance of a permanent magnet synchronous motor," IEEE Trans. Magn., vol. 39, no. 5, pp. 2621-2623, Sep. 2003.

[11] A. E. Fitzgerald and C. Kingsley, Jr., Electric Machinery, 2nd ed. New York: McGraw-Hill, 1961.

[12] J. R. Hendershot and T. J. E. Miller, "Design of Brushless PermanentMagnet Motors," ISBN 1-881855-03-1.

[13] T. J. E. Miller, "PC-BDC and PC-FEA Manuals," SPEED Laboratory, University of Glasgow, 2004.

[14] K. M. Rahman and S. Hiti, "Identification of machine parameters of a synchronous motor," IEEE Trans. Ind. Appl., vol. 41, no. 2, pp. 557-565, Mar.-Apr. 2005.

[15] C. Mademlis and V. G. Agelidis, "On considering magnetic saturation with maximum torque to current control in interior permanent magnet synchronous motor drives," IEEE Trans. Energy Convers., vol. 16, no. 3, pp. 246-252, Sep. 2001.

[16] M. A. Jabbar, H. N. Phyu, Z. Liu, and B. Chao, "Modeling and numerical simulation of a brushless permanent-magnet DC motor in dynamic conditions by time-stepping technique," IEEE Trans. Ind. Appl., vol. 40, no. 3, pp. 763-770, May.-Jun. 2004.

[17] T. J. E. Miller, M. Popescu, C. Cossar, and M. I. McGilp, "Computation of the voltage-driven flux-MMF diagram for saturated PM brushless motors," in IEEE Industry Applications Soc. Annu. Mtg. (IAS), Hong Kong, Oct. 2004, vol. 2, pp. 1023-1028, Paper 05IAS_28P2.

[18] K. Nakamura, K. Saito, and C. Ichinokura, "Dynamic analysis of interior permanent magnet motor based on a magnetic circuit model," IEEE Trans. Magn., vol. 39, no. 5, pp. 3250-3252, Sep. 2003.

[19] J. A. Walker, D. G. Dorrell, and C. Cossar, "Flux-linkage calculation in permanent-magnet motors using the frozen permeabilities method," IEEE Trans. Magn., vol. 41, no. 10, pp. 3946-3948, Oct. 2005.

Manuscript received January 30, 2006; revised March 27, 2006. Corresponding author: M. Popescu (e-mail: mircea@elec.gla.ac.uk, mirceap@ ieee.org). 